\title{
Neuronal GPR30 Participates in Genistein-Mediated Neuroprotection in Ischemic Stroke by Inhibiting NLRP3 Inflammasome Activation in Ovariectomized Female Mice
}

\section{Shiquan Wang}

Air Force Medical University

Zhen Zhang

Air Force Medical University

Jin Wang

Air Force Medical University

Lina Ma

Air Force Medical University

Jianshuai Zhao

Air Force Medical University

Jiajia Wang

Air Force Medical University

Zongping Fang

Air Force Medical University

Wugang Hou

Air Force Medical University

Haiyun Guo (D1532051058@qq.com )

Air Force Medical University https://orcid.org/0000-0003-3897-820X

\section{Research Article}

Keywords: Ischemic stroke, Ovariectomized mice, Genistein, GPR30, NLRP3

Posted Date: December 22nd, 2021

DOI: https://doi.org/10.21203/rs.3.rs-1177656/v1

License: (c) (1) This work is licensed under a Creative Commons Attribution 4.0 International License.

Read Full License 


\section{Abstract}

Estrogen replacement therapy (ERT) is potentially beneficial for the prevention and treatment of postmenopausal cerebral ischemia but inevitably increases the risk of cerebral hemorrhage and breast cancer when used for a long period of time. Genistein, a natural phytoestrogen, has been reported to contribute to the recovery of postmenopausal ischemic stroke with reduced risks. However, the underlying mechanism of genistein-mediated neuroprotection remains unclear. We reported that genistein exerted significant neuroprotective effects by enhancing the expression of neuronal $\mathrm{G}$ protein-coupled receptor 30 (GPR30) in the ischemic penumbra after cerebral reperfusion in ovariectomized (OVX) mice, and this effect was achieved through GPR30-mediated inhibition of nod-like receptor protein 3 (NLRP3) inflammasome activation. In addition, we found that Peroxisome proliferator-activated receptor-gamma coactivator 1a (PGC-1a) was the pivotal molecule that participated in GPR30-mediated inhibition of NLRP3 inflammasome activation in OVX mice after ischemia/reperfusion (I/R) injury. Our data suggest that the neuronal GPR30/PGC-1 a pathway plays an important role in genistein-mediated neuroprotection against I/R injury in OVX mice.

\section{Introduction}

Postmenopausal stroke seriously threatens health and shortens life expectancy in women[1, 2]. How to effectively reduce cerebral ischemic injury in postmenopausal women and improve patient prognosis is an urgent scientific problem to be solved. A previous study suggested that the rising morbidity and disability rates of cerebral stroke in postmenopausal women are mainly due to estrogen deficiency, and ERT works as a promising preventive and therapeutic strategy[3-5]. However, a series of studies showed that ERT not only significantly increases the incidence of endometrial cancer and breast cancer in postmenopausal women, but also had a time window for its neuroprotective effect, which seriously impairs the clinical application of ERT for stroke prevention[6-8]. Therefore, estrogen-like substances are urgently needed and should receive more attention for postmenopausal stroke therapy.

Genistein (4',5,7-trihydroxyisoflavone) is one of the most abundant phytoestrogens and can be extracted from plants such as soybeans and broad beans[9]. Recent studies support the benefits of genistein in protecting the brain from chronic or acute damage through involvement in inflammatory responses, apoptotic pathways and oxidative stress[10]. Moreover, genistein may serve as an alternative therapy for postmenopausal patients with cerebral ischemia due to the lack of ERT-related side effects[11]. However, the underlying targets of genistein-mediated neuroprotection remain largely unknown.

GPR30, an estrogen membrane receptor, is widely expressed in the nervous system[12]. Our previous studies showed that GPR30 activation alleviated I/R injury in OVX mice by inhibiting the microglial inflammatory response[13]. Inflammation plays a crucial role in ischemic stress by affecting the survival of neuronal cells, and inflammasomes have attracted increasing attention because of their ability to trigger inflammation[14, 15]. As the most widely reported inflammasome in the brain, the NLRP3 inflammasome can be activated by endogenous stress and injury signals[14, 15]. Moreover, our latest 
study revealed that genistein alleviated neurological deficits after ischemia in reproductively senescent mice by inhibiting microglial NLRP3 expression[17]. However, there is still no conclusive evidence to verify whether the anti-inflammatory effect of genistein after I/R injury is mediated by GPR30.

PGC-1a, a key transcriptional integrator, is involved in multiple physiological functions, especially the generation of proinflammatory mediators $[18,19]$. In addition, PGC-1 a might be activated by exogenous neuroprotective stimuli, such as exercise[20]. Notably, a recent study suggested a reduction in NLRP3 activation in microglia-specific PGC-1a-overexpressing mice[21]. Therefore, we hypothesized that genistein may indirectly regulate the activation of the NLRP3 inflammasome by acting on GPR30 and that PGC-1 a may play an intermediate regulatory role in the GPR30/NLRP3 pathway.

To confirm the role of GPR30 as a promoter of the neuroprotective effects of genistein, we first examined the temporal expression of GPR30 and assessed cerebral infarct size, neurobehavioral deficits, and apoptosis after the downregulation of neuronal GPR30. Then, to investigate the role of the NLRP3 inflammasome in genistein-mediated neuroprotection, we examined inflammasome signaling in the ischemic penumbra after neuronal GPR30 downregulation, as well as inflammasome-mediated apoptosis. Furthermore, to explore the role of PGC-1a in mediating the GPR30/NLRP3 pathway, we examined the activation of the NLRP3 inflammasome and ischemia-induced brain injury following the downregulation of neuronal PGC-1a. Our data reveal that neuronal GPR30 is a promising therapeutic target for postmenopausal cerebral ischemia.

\section{Materials And Methods}

\section{Animals and groups}

Eight-week-old female C57BL/6J mice were provided by the Experimental Animal Center of the Fourth Military Medical University. The mice were housed at $22 \pm 2{ }^{\circ} \mathrm{C}$ and $50 \pm 1 \%$ relative humidity. All animal studies (including mouse euthanasia procedures) were conducted in accordance with the guidelines of the Animal Care Committee of the Fourth Military Medical University and in compliance with the guidelines of the AAALAC and IACUC. The mice were randomly assigned to different experimental groups, and all surgeries and behavioral tests were performed by investigators who were blinded to the groups.

First, all mice were subjected to ovariectomy. After 1 week, the mice were randomly assigned to the following groups: Sham, MCAO, Vehicle and Gen groups. Except for those in the Sham group, the other mice underwent unilateral middle cerebral artery occlusion (MCAO). Genistein (10 mg/kg) was intraperitoneally injected into Gen group mice at $6 \mathrm{~h}, 24 \mathrm{~h}$ and $48 \mathrm{~h}$ after MCAO as previously described[17]. The protein and mRNA levels of GPR30 were measured at $3 \mathrm{~d}$ after MCAO.

Then, we used hSyn-GPR30-shRNA to knockdown neuronal GPR30 to examine the effect of GPR30 on genistein-induced neuroprotection and NLRP3 inflammasome pathway inhibition during cerebral I/R. All ovariectomized animals were assigned into one of six groups: Sham, MCAO, Vehicle, Gen, Control virus 
and GPR30 virus groups. The first four groups were the same as described above. Mice in the control virus and GPR30 virus groups were subjected to MCAO 3 weeks after virus injection and were treated with genistein postoperatively. In addition, to explore whether PGC-1a is a key target in GPR30mediated inhibition of NLRP3 inflammasome activation, we used hSyn-PGC-1a-shRNA to knockdown neuronal PGC-1a. The ovariectomized animals were divided into the control virus and PGC-1a virus groups. All animals were subjected to MCAO and intraperitoneal injection of genistein.

Infarct volume and TUNEL staining were analyzed at $7 \mathrm{~d}$ after reperfusion. The protein and mRNA levels of NLRP3 inflammasome-related proteins and PGC-1a were measured at $3 \mathrm{~d}$ after I/R. Inflammatory cytokines were measured at $3 \mathrm{~d}$ after I/R.

\section{Drugs Dilutions and Treatment}

Genistein was purchased from Selleck Chemicals (Houston, Texas, USA), dissolved in dimethyl sulfoxide (DMSO) to a concentration of $200 \mathrm{mg} / \mathrm{ml}$, then diluted with mixed liquid (saline $50 \%$, PEG40\% and tween$8010 \%$ ) to a concentration of $1 \mathrm{mg} / \mathrm{ml}$ (DMSO was $0.5 \%$ ). Mice were intraperitoneally injected with (10 $\mathrm{mg} / \mathrm{kg}$ ) genistein or an equivalent solvent at $6 \mathrm{~h}, 24 \mathrm{~h}$ and $48 \mathrm{~h}$ after reperfusion.

\section{Ovariectomy}

Ovariectomy was performed with bilateral dorsolateral incisions on the abdominal frontal wall to remove both ovaries[22]. The efficacy of ovariectomy was evaluated by vaginal cytology of mice for 7 consecutive days to confirm the cessation of the estrus cycle[23].

\section{Focal cerebral ischemia/reperfusion}

MCAO was performed as described previously[24]. In brief, the mice underwent $1.5 \%$ isoflurane anesthesia, a small incision was made in the skin of the right neck and the muscle was separated under a microscope to reveal the right common carotid artery. An incision is then made in the right external carotid artery, where silicon-coated suture (RWD Life Science) was placed and passed forward through the internal carotid artery to block the middle cerebral artery. After $60 \mathrm{~min}$, the silicon-coated suture was withdrawn, and the wound was sewed up. A heating pad was used throughout the operation, and the temperature of the anus was monitored, which was maintained at $36.5-37.5^{\circ} \mathrm{C}$. A laser speckle contrast imager was used to record cortical blood flow, which estimated intravascular RBC movement from an image stack after a $6 \mathrm{~ms}$ exposure time and was programmed to generate 9 pixels $(3 \times 3)$ for comparison calculations. Each mouse was measured three times: 10 min before MCAO, during MCAO and 10 min after MCAO. Only animals whose local cerebral blood flow was reduced by more than $80 \%$ of the preMCAO baseline level were included in further experiments. Representative laser speckle imaging is shown in Fig. S1. Those animals that died during or immediately after surgery (excluded from the study) had a mortality rate of about 10 percent. Sham-operated animals underwent the same anesthesia and surgery except for MCA occlusion. 
The ischemic penumbra was defined and sampled as previously described. In short, a coronal section was taken from the anterior tip of the frontal lobe $(2 \mathrm{~mm}$ to $6 \mathrm{~mm}$ ) (4-mm thick), followed by a longitudinal section (top to bottom) of the right hemisphere approximately $1 \mathrm{~mm}$ from the midline. Then, a cut was made at approximately 2 o'clock from the midpoint of the section, and the wedged tissue was the penumbra.

\section{Measurement of infarct size}

Mice were transcardially perfused with precooled normal saline immediately after anesthesia. The brain was removed and sliced into $1 \mathrm{~mm}$ coronal slices. These sections were then incubated at $37^{\circ} \mathrm{C}$ in a $2 \%$ solution of 2,3,5-triphenyltetrazolium chloride (TTC, Sigma) for $15 \mathrm{~min}$ and fixed in $4 \%$ paraformaldehyde (PFA) overnight. Image analysis software was used to compute the infarct size after the stained brain tissue was photographed. The infarct ratio was computed with the following formula: infarct volume $=$ (contralateral hemisphere area -ipsilateral hemisphere noninfarct area)/contralateral area.

\section{Assessment of behavioral deficits}

Neurobehavioral outcomes were measured by the grid-walking test, the adhesive removal test and the neurological deficit score. For the first two tests, mice were given 3 consecutive days of training before the operationto obtain a stable baseline value, and behavioral tests were performed at 1, 3 and $7 \mathrm{~d}$ after I/R.

As described in a previous study[25], the adhesive removal test can be used to evaluate somatosensory deficits. After the mice were acclimated in the test chamber for $5 \mathrm{~min}$, tape $(0.3 \times 0.4 \mathrm{~cm})$ was pasted on the left or right forepaws with equal pressure. The time until removal of the tape from each forepaw was assessed. The maximum testing time was $120 \mathrm{~s}$. If this time was exceeded, it was recorded as $120 \mathrm{~s}$.

The grid-walking test is used to evaluatethe walking performance of mice. The grid apparatus $(40 \times 20$ $\mathrm{cm}^{2}$; grid cell: $2 \times 2 \mathrm{~cm}^{2}$; height: $50 \mathrm{~cm}$ ) was located in a sound attenuated room. Performance was recorded for $60 \mathrm{~s}$ using a video camera located beside the apparatus at an angle of approximately 20 to 40 degrees. A foot slip was recorded when one paw completely missed a bar, with the limb falling between the bars, or when the paw was correctly placed on the bar but slipped off during weight bearing. The total steps of the left forelimb and hind limb were counted, and the foot fault ratio was determined by dividing the number of foot slips of the left forelimb and left hind limb by the total number of left steps taken within $60 \mathrm{~s}$.

The neurological deficit score was based on the previously described scoring system and was assessed blind at 7 days after $\mathrm{I} / \mathrm{R}[26]$. It includes six tests including spontaneous activity, symmetry of movements, symmetry of forelimbs, climbing wall of wire cage, reaction to touch on either side of trunk, and response to vibrissa touch. The scores indicate the following: for the first three tests, no movement (0), slight movement (1), slow movement (2), and normal movement (3); for the last three tests, no movement or response (1), weak movement or response (2), and normal movement or response (3). Each mouse's 
score after completing the assessment was the sum of all six separate test scores. The neurological deficit score ranged from 3 to 18 . The higher the score, the better the neurological outcome.

\section{Immunofluorescence staining and TUNEL staining}

After 7 days of reperfusion, the mice were anesthetized with isoflurane and then transcardially perfused with normal saline followed by $4 \%$ PFA. The brain was removed and fixed in $4 \%$ PFA at $4{ }^{\circ} \mathrm{C}$ overnight and then dehydrated in $30 \%$ sucrose until the tissue completely subsided. Then, $12 \mu \mathrm{m}$ thick coronal sections were prepared $(0.5 \mathrm{~mm}$ prior to bregma). Five sections were randomly selected from each mouse for staining, washed three times using PBS, permeabilized with $0.3 \%$ Triton X-100-PBS for 1 $\mathrm{h}$, blocked in $10 \%$ donkey serum for $1 \mathrm{~h}$, and then incubated with primary antibodies overnight at $4{ }^{\circ} \mathrm{C}$. The following primary antibodies were used: mouse anti-NLRP3 (1:100, Adipogen), guinea pig anti-NeuN (1:300, Millipore), rabbit anti-GPR30 (1:100, Abcam), and rabbit anti-PGC-1a. Then, the sections were incubated with secondary antibodies (Alexa Fluor 488-nm donkey anti-guinea pig IgG, Alexa Fluor 594-nm donkey anti-rabbit IgG, Alexa Fluor 488-nm donkey anti-rabbit $\lg G$ ) at room temperature for $2 \mathrm{~h}$ in the dark. Finally, five fields of the penumbra zone in each section were photographed randomly with the 40x objective lens on an Olympus (U-HGLGPS Japan) fluorescence microscope. In addition, an in situ cell death detection kit was used to examine TUNEL staining (Roche Diagnostics, Mannheim, Germany). The apoptosis index was calculated as the ratio of TUNEL and NeuN double-positive cells to NeuN-positive cells.

\section{Duolink 『 proximity ligation assay}

The washing, permeabilizing and blocking processes of the Duolink 『in situ proximity ligation assay (PLA) immunoassay were similar to those of immunofluorescence staining. Briefly, brain tissue sections were incubated with anti-NLRP3 (1:100, Adipogen) and anti-ASC (1:100, Abcam) antibodies at $4{ }^{\circ} \mathrm{C}$ overnight. After being washed, the sections were then incubated with Duolink PLA Rabbit MINUS and PLA Goat PLUS proximity probes at $37^{\circ} \mathrm{C}$ for $2 \mathrm{~h}$. Ligation and amplification were performed using the Duolink in situ detection reagent kit. DAPI was used to counterstain the nuclei. An Olympus (Japan) fluorescence microscope was used to capture images, and red dots indicate interactions between NLRP3 and ASC.

\section{Western blotting}

Western blotting was performed according to our previous studies[24]. The antibodies used were as follows: mouse anti-NLRP3 (1:1000, Adipogen), rabbit anti-pro-caspase-1 (1:1000, Santa Cruz), mouse anti-cleaved caspase-1 (1:3000, Adipogen), rabbit anti-GPR30 (1:1000, Abcam), rabbit anti-PGC-1a (1:1000, Abcam), rabbit anti-GAPDH (1:1000, Cell Signaling Technology), and secondary anti-rabbit or mouse antibodies (1:10000, Thermo Scientific).

\section{Quantitative reverse transcription polymerase chain reaction (RT-qPCR)}


According to the instructions, TRIzol reagent (Life Technologies, USA) was used to obtain total RNA in the ischemic penumbra. Then, a standard cDNA synthesis kit (Life Technologies, USA) was used for cDNA synthesis. The mRNA level of $\beta$-actin was used as an internal control. The primer sequences were as follows: GPR30 forward: 3'-AACAGAGCAGCGATCTGGAC-5', reverse: 5'-GCAGAGTCCTTGGATGGCTT-3'; PGC-1 a forward: 3'-AGCCGTGACCACTGACAACGAG-5', reverse: 5'-GCTGCATGGTTCTGAGTGCTAAG-3'; GAPDH forward: 3'-AACAGAGCAGCGATCTGGAC-5', reverse: 5'-GCAGAGTCCTTGGATGGCTT-3'; NLRP3 forward: 3'-GCTGTGTGAGGCACCAG-5', reverse: 5'-GGAGATGTCGAAGCAGCATT3'; and Caspase-1 forward: 3'-GCTGTGTGAGGCACCAG-5', reverse: 5'-GCATAGGTCTAAGAATGAACTGGA3'. The reverse transcription reaction was performed in a $20 \mu \mathrm{l}$ volume with $500 \mathrm{ng}$ of total RNA at $16^{\circ} \mathrm{C}$ for $30 \mathrm{~min}, 42$ ${ }^{\circ} \mathrm{C}$ for $42 \mathrm{~min}$, and $85^{\circ} \mathrm{C}$ for $5 \mathrm{~min}$. PCR cycling started with template denaturation at $95^{\circ} \mathrm{C}$ for $5 \mathrm{~min}$, followed by 40 cycles of $95^{\circ} \mathrm{C}$ for $10 \mathrm{~s}, 60^{\circ} \mathrm{C}$ for $20 \mathrm{~s}, 72{ }^{\circ} \mathrm{C}$ for $20 \mathrm{~s}$, and $78^{\circ} \mathrm{C}$ for $20 \mathrm{~s}$.

\section{Evaluation of inflammatory cytokines}

The penumbra tissues were homogenized by ultrasound and centrifuged at 10,000 $\mathrm{g}$ for $15 \mathrm{~min}$, and the supernatant was collected and cryopreserved at $-80^{\circ} \mathrm{C}$. ELISA kits (Nanjing Jianchen Bioengineering Institute) were used to measure inflammatory factors (TNF-a, IL-1 $\beta, I L-6, I L-18)$, and the assay was performed strictly in accordance with the manufacturer's protocols.

\section{Transfection of AAV}

AAV containing hSyn-PGC-1 a-shRNA-GFP or hSyn-GPR30-shRNA-GFP was purchased from GeneChem Co., Ltd. (Shanghai, China). The target sequence of PGC-1a was 5'CCUGUUUGAUGACAGCGAA-3', and the target sequence of GPR30 was 5'-CATGTACAGCAGCGTCTTC-3'. Transfection was performed by stereotactic injection. The injection coordinates were $1.5 \mathrm{~mm}$ lateral to the midsagittal line, $0.4 \mathrm{~mm}$ anterior to the bregma, and $1.5 \mathrm{~mm}$ deep in the dura mater. Three weeks after injection, the presenceof AAV was determined by immunofluorescence analysis and Western blotting. The results are shown in Fig. S2.

\section{Statistical analysis}

Statistical analyses were carried out using GraphPad Prism 7.0 software. All data are presented as the mean \pm standard deviation. Independent t-tests were used to analyze differences between two independent groups. One-way ANOVA followed by the Newman-Keuls test was used for multiple groups. Repeated measures analysis was used to assess differences between groups in the neurobehavioral tests. $\mathrm{P} \otimes 0.05$ was considered statistically significant.

\section{Results}

\section{Genistein reversed the downregulation of neuronal GPR30 expression induced by I/R injury}


We first measured the temporal expression of GPR30 in the ischemic penumbra after reperfusion in OVX mice. Western blot analysis revealed a time-dependent decrease in GPR30 expression after I/R compared with that in the sham group (Fig. 1a). GPR30 expression was prominently decreased at $1 \mathrm{~d}$ after I/R, reached the lowest point at $3 \mathrm{~d}$, and remained reduced for at least $14 \mathrm{~d}$. In addition, the mRNA level of GPR30 was also decreased in OVX mice after I/R and reached the lowest point at $3 \mathrm{~d}$ (Fig. 1c).

Next, we examined the effect of genistein on the expression of GPR30 after I/R injury. Genistein was administered intraperitoneally to OVX mice at $6 \mathrm{~h}, 24 \mathrm{~h}$ and $48 \mathrm{~h}$ after l/R. Considering that the protein and mRNA levels of GPR30 decreased most significantly at $3 \mathrm{~d}$ after reperfusion, samples were collected at $3 \mathrm{~d}$ after reperfusion for analysis. As shown in Fig. 1b, genistein notably increased the expression of GPR30 in the penumbra compared with that in the vehicle group at $3 \mathrm{~d}$ after reperfusion. Furthermore, the transcriptional level of GPR30 was increased in OVX mice after cerebral I/R (Fig. 1d). GPR30 has been indicated to be widely expressed in neurons, microglia and astrocytes, and we then investigated the cell specificity of GPR30 using double immunofluorescence staining. The results showed that the percentage of $\mathrm{GPR} 30^{+} \mathrm{NeuN}^{+}$cells relative to GPR30+ cells in the penumbra region reached about $70 \%$, indicating that GPR30 was mainly expressed in neurons (Fig. 1e and 1f). In addition, genistein significantly increased the expression of GPR30 in neurons in the ischemic penumbra (Fig. 1g). Accordingly, these results suggest that I/R stress can downregulate the protein and mRNA levels of GPR30 in neurons, and this effect is reversed by genistein administration.

\section{GPR30 plays an important role in genistein-mediated defense against neurologic deficits and apoptosis after ischemic injury}

To determine whether GPR30 is involved in the neuroprotective effect of genistein on ischemia, we constructed an in vivo model of neuron-specific GPR30 knockdown using AAVs (Fig. S2a-2c). Then, for GPR30 down-regulated mice, whether treatment with genistein can improve the prognosis of ischemia? The results revealed that the infarct volumes of genistein-treated OVX mice were notably decreased at $7 \mathrm{~d}$ after I/R, and this effect was blocked by GPR30 downregulation (Fig. 2a and 2b). In addition, the adhesive removal test was used to assess sensomotor dysfunction, and the time to removal was decreased in the genistein administration group (Fig. 2c). We also conducted a grid-walking test to examine the level of motor deficits, and the results revealed that genistein significantly increased the total steps and greatly reduced the foot fault ratio in OVX mice after I/R (Fig. $2 \mathrm{~d}$ and 2e). Importantly, virus-mediated downregulation of neuronal GPR30 expression in the penumbra before MCAO exacerbated sensorimotor injury compared to the control virus group, even after postoperative treatment with genistein (Fig. 2c-2e).

Our previous study showed that genistein had a significant antiapoptotic effect [17]. Here, we also investigated the impact of regulating GPR30 on the antiapoptotic effect of genistein. The data illustrated that genistein reduced the number of TUNEL-positive neurons in the penumbra, while the reduction in apoptosis induced by genistein disappeared after the downregulation of GPR30 (Fig. 3a and 3b). The protein level of cleaved caspase 3, a well-known apoptotic executor, was significantly decreased in the genistein group. However, there was a noticeable increase in the GPR30 virus group compared to the 
control virus group, even though both groups were treated with genistein (Fig. 3c). Overall, these results suggest that GPR30 participates in genistein-mediated neuroprotection in the ischemic penumbra after cerebral reperfusion. It is worth noting that the neurological outcomes and apoptosis in the GPR30 virus group were worse than those in the control virus group, but there was still a statistical difference compared with that in the vehicle group (Fig. $2 b$ and $3 b$ ), indicating that neuronal GPR30 was only partially involved in the neuroprotective effect of genistein, and there may be other molecular pathways.

\section{GPR30 participated in genistein-mediated neuroprotection by suppressing the synthesis and activation of the NLRP3 inflammasome}

To uncover the relationship between GPR30 and the NLRP3 inflammasome, we first determined the effect of genistein treatment on NLRP3 protein levels after I/R. Western blot analysis indicated that genistein greatly decreased NLRP3 expression in the ischemic penumbra at $3 \mathrm{~d}$ after reperfusion, while the expression of NLRP3 was further increased after the downregulation of GPR30 (Fig. 4a). Additionally, the mRNA level of NLRP3 was also decreased by genistein treatment and increased by GPR30 deficiency (Fig. 4c). Furthermore, double immunofluorescence staining showed that the expression of neuronal NLRP3 was downregulated by genistein and upregulated by GPR30 deficiency (Fig. 4e-4g). NLRP3 can recruit pro-caspase-1 to form an inflammasome complex with ASC to activate caspase-1. We also measured the level of pro-caspase-1 and found that genistein could suppress the expression of procaspase-1 at $3 \mathrm{~d}$ after I/R, and this effect could be blocked by GPR30 downregulation (Fig. 4b), which was consistent with the change in the mRNA level of pro-caspase-1 (Fig. 4d).

Given that the binding of ASC is critical for NLRP3 inflammasome activation, we next examined the interaction between these two proteins using the Duolink II proximity ligation assay. Duolink puncta (red) indicated the interaction of NLRP3 with ASC. Quantitative analysis indicated that genistein notably reduced the number of Duolink puncta compared with that in the vehicle group of OVX mice at $3 \mathrm{~d}$ after I/R and that GPR30 downregulation increased the number of Duolink puncta (Fig. $5 \mathrm{a}$ and $5 \mathrm{~b}$ ). Activated caspase-1 in the NLRP3 inflammasome cleaves the cytokine precursors pro-interleukin-1 beta (pro-IL-1 $\beta$ ) and pro-IL-18 into their mature forms: IL-1 $\beta$ and IL-18 [27]. Thus, we examined caspase-1, IL-1 $\beta$, IL-18 and two other inflammatory cytokines (tumor necrosis factor alpha (TNF-a) and IL-6) in the ischemic penumbra at $3 \mathrm{~d}$ after cerebral reperfusion. The results revealed that the levels of caspase-1, IL-1 $\beta$, IL-18, TNF- $\alpha$ and IL- 6 in the genistein group were strongly reduced, and as expected, GPR30 downregulation reversed the genistein-induced decrease in these inflammatory cytokines in OVX mice. (Fig. $5 \mathrm{c}-5 \mathrm{~g}$ ). In conclusion, we showed that GPR30 mediated the anti-inflammatory effect of genistein by indirectly inhibiting the activation of the NLRP3 inflammasome. However, the molecular pathway by which GPR30 inhibits the activation of NLRP3 inflammasomes is unknown.

\section{GPR30 suppressed the formation of the NLRP3 inflammasome by elevating the expression of PGC-1a}

Peroxisome proliferator-activated receptor-gamma coactivator-1a (PGC-1a) is a key coregulator of gene transcription and is involved in many neurological diseases, such as neurodegeneration and neuroinflammation. To verify whether PGC-1 a participates in GPR30-mediated inhibition of the NLRP3 
inflammasome in genistein-induced neuroprotection in cerebral I/R injury, we first measured the expression of PGC-1a in the genistein-treated I/R model. We found that PGC-1a expression was upregulated in both cytoplasm and nucleus after reperfusion, and further upregulated after genistein supplementation. However, PGC-1a was suppressed by GPR30 knockdown (Fig. 6a and 6b). Additionally, the mRNA level of PGC-1a was also increased in the genistein group and further decreased in the GPR30knockdown group (Fig. 6c). Double immunofluorescence staining was performed and further showed that PGC-1a expression was increased in neurons treated with genistein and that GPR30 knockdown blocked the genistein-induced increase in PGC-1a in OVX mice (Fig. 6d and 6e). These results suggest that PGC1a may be an endogenous protective molecule and a regulated target of GPR30 in genistein neuroprotective effect.

Next, we constructed a virus that specifically downregulated neuronal PGC-1a to observe the effect of PGC-1a downregulation on the synthesis and activation of the NLRP3 inflammasome in genisteinmediated neuroprotection against I/R injury. We found that neuronal PGC-1a downregulation increased the protein and mRNA levels of NLRP3 after genistein administration in OVX mice during I/R injury (Fig. 7b and 7d). Immunofluorescence staining further showed that neuron-specific PGC-1a downregulation could increase NLRP3 expression levels in neurons (Fig. 7a, $7 \mathrm{f}$ and 7g). In addition, procaspase-1 protein and mRNA levels were also increased in the PGC-1 a virus group, which was consistent with the changes in NLRP3 (Fig. 7c and 7e). Furthermore, we confirmed that neuronal PGC-1a downregulation after treatment with genistein could increase the number of NLRP3-ASC complexes and the activation of caspase-1 (Fig. 8a-8c) and promote the release of inflammatory cytokines (Fig. $8 \mathrm{~d}-8 \mathrm{~g}$ ) in the penumbra at $3 \mathrm{~d}$ after I/R. All of these suggest that PGC-1a regulates the synthesis and activation of NLRP3 inflammasome and the release of downstream inflammatory cytokines.

Moreover, we further verified whether regulation of PGC-1a affects the neuroprotective effect of genistein, and found that the infarct volumes in the PGC-1 a knockdown group were significantly higher than those in the control virus group (Fig. 9a and 9b). We then assessed sensorimotor deficits following the downregulation of neuronal PGC-1 a using the adhesive removal and grid-walking tests. The time taken to remove the adhesive tape was clearly increased in the PGC-1a-knockdown group (Fig. 9c). In addition, PGC-1 a downregulation resulted in obvious decreases in the total steps and increases in the foot fault ratio in the grid-walking test after the restoration of circulation (Fig. 9d and 9e). TUNEL staining revealed that PGC-1 a downregulation accelerated neuronal apoptosis at $7 \mathrm{~d}$ after reperfusion (Fig. $9 \mathrm{f}$ and $9 \mathrm{~g}$ ). Caspase-3 analysis also showed that PGC-1 a downregulation exacerbated neuronal apoptosis after I/R (Fig. 9h). These results indicate that PGC-1a in neurons contributes to the neuroprotective effect as an intermediate regulator and mediates the regulatory effect of GPR30 on the NLRP3 inflammasome, but do not rule out the existence of other neuroprotective pathways.

\section{Discussion}

This study offers several new discoveries. First, genistein had significant anti-inflammatory and neuroprotective effects on the ischemic penumbra after I/R by upregulating neuronal GPR30. Second, 
GPR30 knockdown counteracted the suppression of NLRP3 activation and inflammatory cytokines production during genistein-mediated neuroprotection against reperfusion injury. Third, neuronal PGC-1a functions as an important mediator between GPR30 and NLRP3 activation during I/R injury. These findings suggest that the GPR30/PGC-1a/NLRP3 pathway is a promising target for alleviating cerebral I/R (Fig. 10).

Genistein is a natural isoflavone that is used as an alternative to postmenopausal hormone therapy for brain disease due to its safety and ability to effectively penetrate the blood-brain barrier (BBB) penetration $[9,10,28]$. Some preclinical studies support the beneficial effects of genistein in protecting against ischemia or other brain damage[29,30]. It is thought that, like estrogen, genistein is a multipotent molecule that is involved in a variety of distinct mechanisms to strengthen brain recovery, including reducing oxidative stress, promoting growth factor signaling, and immunosuppression[31]. Additionally, these effects occur in endothelial cells, glia, and neurons to provide a synergistically beneficial profile to fight against brain injury[10]. However, the underlying mechanism of genistein-mediated neuroprotection against I/R injury is not fully understood, which is a serious question that remains to be solved.

GPR30 is widely located in the hippocampus, cortex, striatum and other nervous system regions. Previous studies on genistein have mainly focused on classic nuclear estrogen receptors[32, 33], while the effect of genistein on GPR30 has been less well studied. A recent study demonstrated that GPR30 participated in the anti-inflammatory effect of genistein on LPS-induced microglial activation[34]. In this study, we observed that the downregulation of GPR30 expression in the ischemic penumbra induced by MCAO/R could be partially reversed by genistein in OVX mice. Importantly, the upregulated GPR30 was mainly expressed in neurons. However, the precise role of neuronal GPR30 in genistein-mediated neuroprotection has not been clarified, especially with regard to the specific downstream signaling pathways. To uncover the exact role of GPR30 in neurons, we constructed a neuron-specific GPR30 downregulation model in vivo and further examined the anti-inflammatory and antiapoptotic effects of genistein. The results revealed that neuronal GPR30 participates in genistein-induced neuroprotection against brain damage, and its underlying mechanism requires more attention.

Neuroinflammation also significantly contributes to neuronal death in reperfusion injury after ischemic stroke[35]. The NLRP3 inflammasome is a major inflammatory pathway that regulates caspase-1 activation and triggers the release of the proinflammatory cytokines IL-1 $\beta$ and IL-18[27]. Our previous study confirmed that genistein could inhibit the NLRP3 inflammasome in microglia and exert antiinflammatory effects. However, the correlation between GPR30 and the NLRP3 inflammasome and its potential key molecules, especially in neurons, are still poorly understood. Here, we showed that neuronal GPR30 upregulation induced by genistein could inhibit the activation of the NLRP3 inflammasome. Furthermore, we discovered that genistein dramatically decreased inflammatory cytokines, and this effect was reversed by the downregulation of GPR30 after I/R injury. The maturation of IL-1 $\beta$ and IL-18 is mainly regulated by the NLRP3 inflammasome, which is responsible for many inflammatory diseases[36]. We hypothesized that genistein plays an anti-inflammatory role by upregulating the transcription and expression of GPR30, thereby indirectly inhibiting the activation of the NLRP3 inflammasome. 
A recent study indicated that microglial PGC-1 a plays a key role in protecting against cerebral ischemic damage by reducing NLRP3 activation and proinflammatory cytokine production[21]. PGC-1a is a key coregulator of gene transcription and is involved in the occurrence of a variety of neurological diseases, such as neurodegeneration and neuroinflammation[37]. Whether PGC-1a is involved in the signaling pathway by which GPR30 regulates the NLRP3 inflammasome remains unclear. To this end, we specifically designed an in vivo model of neuron-specific PGC-1 a knockdown by AAV transfection. Surprisingly, we observed that PGC-1a expression in the penumbra was upregulated after ischemia reperfusion, but it was more significantly upregulated in the genistein administration group. Importantly, the downregulation of neuronal GPR30 antagonized the genistein-induced increase in PGC-1a. Moreover, we also found that neuronal PGC-1 a downregulation increased the synthesis and activation of NLRP3 inflammasomes and exacerbated neurological dysfunction and apoptosis after I/R injury. These results strongly indicate that neuronal PGC-1 plays a pivotal role in genistein-mediated neuroprotection.

It should be pointed out that in our study, the down-regulation of neuronal GPR30 only partially reversed the neuroprotective effect induced by genistein, suggesting that there may be other molecular pathways other than GPR30, which is consistent with previous studies. For example, Donzelli et al. demonstrated that genistein exerts a neuroprotective effect by activating ER $\beta$ in cerebral ischemia models[32]. Studies have shown that genistein can reduce nerve damage by targeting oxidative stress, apoptosis and inflammation[10]. There is increasing evidence that genistein is a pleiotropic neuroprotective molecule that acts on a variety of receptors, cell types, signaling pathways and genes.

This pathway illuminates the underlying mechanism of GPR30-induced inflammation in neurons during reperfusion injury after ischemic stroke. In conclusion, this study provides a new idea for the underlying mechanism behind the neuroprotective effect of genistein on cerebral ischemia/reperfusion injury. Neuronal GPR30 upregulation induced by genistein treatment can inhibit the activation of NLRP3 inflammasomes and result in anti-inflammatory and antiapoptotic effects on reperfusion by upregulating PGC-1a. Therefore, our study demonstrates that neuronal GPR30 enhances the endogenous defense system of neurons in the penumbra to fight against brain damage after I/R injury.

\section{Abbreviations}

\section{ERT}

Estrogen replacement therapy

GPR30

G protein-coupled receptor 30

OVX

Ovariectomized

NLRP3

Nod-like receptor protein 3

PGC-1a

Peroxisome proliferator-activated receptor-gamma coactivator $1 \mathrm{a}$ 
Genistein

4',5,7-Trihydroxyisoflavone

I/R

Ischemia/reperfusion

MCAO

Middle cerebral artery occlusion

TTC

2,3,5-Triphenyltetrazolium chloride

PFA

Paraformaldehyde

PBS

Phosphate-buffered saline

TUNEL

Terminal deoxynucleotidyl transferase-mediated dUTP nick-end labeling

GAPDH

Glyceraldehyde 3-phosphate dehydrogenase

RT-qPCR

Quantitative reverse transcription polymerase chain reaction

PLA

Proximity ligation assay

ASC

Apoptosis-associated speck-like protein.

\section{Declarations}

Funding This work was supported by the National Natural Science Foundation of China (no. 81971226 to WGH; no. 81901079 to HYG), the Shaanxi Provincial Natural Science Foundation for Distinguished Young Scholars (no. 2021JC-33 to WGH) and the Surface Project of Shaanxi Provincial Natural Science Foundation (no. 2030JM-330 to SQW).

Competing interests The authors declare that they have no competing interests.

Author contributions H.G. and W.H. conceived and designed the study. S.W., Z.Z., J.W., L.M., J.Z., J.W. and Z.F. performed the experiments and data analysis. S.W., Z.Z. and J.W. prepared figures and the manuscript draft. H.G. wrote the paper. All authors read and approved the final manuscript.

Data Availability All data generated or analyzed during this study are included in this published article and its supplementary information files.

Ethics approval All institutional and national guidelines for the care and use of laboratory animals were followed. 
Consent to participate Not applicable.

Consent to publish Not applicable.

Acknowledgements Not applicable.

\section{References}

1. Persky RW, Turtzo LC, Mccullough LD (2010) Stroke in women: disparities and outcomes. Curr Cardiol Rep 12(1):6-13. https://10.1007/s11886-009-0080-2

2. Reeves MJ, Bushnell CD, Howard G, Gargano JW, Duncan PW, Lynch G, Khatiwoda A, Lisabeth L (2008) Sex differences in stroke: epidemiology, clinical presentation, medical care, and outcomes. Lancet Neurol 7(10):915-926. https://10.1016/S1474-4422(08)70193-5

3. Alonso DLM, Egido JA, Fernandez C, Martinez-Vila E, Santos S, Morales A, Martinez E, Pareja A et al (2007) Risk of ischemic stroke and lifetime estrogen exposure. Neurology 68(1):33-38. https://10.1212/01.wnl.0000250238.69938.f5

4. Grady D, Rubin SM, Petitti DB, Fox CS, Black D, Ettinger B, Ernster VL, Cummings SR (1992) Hormone therapy to prevent disease and prolong life in postmenopausal women. Ann Intern Med 117(12):1016-1037. https://10.7326/0003-4819-117-12-1016

5. Engler-Chiurazzi EB, Brown CM, Povroznik JM, Simpkins JW (2017) Estrogens as neuroprotectants: Estrogenic actions in the context of cognitive aging and brain injury. Prog Neurobiol 157:188-211. https://10.1016/j.pneurobio.2015.12.008

6. Beral V (2003) Breast cancer and hormone-replacement therapy in the Million Women Study. Lancet 362(9382):419-427. https://10.1016/s0140-6736(03)14065-2

7. Rossouw JE, Anderson GL, Prentice RL, Lacroix AZ, Kooperberg C, Stefanick ML, Jackson RD, Beresford SA et al (2002) Risks and benefits of estrogen plus progestin in healthy postmenopausal women: principal results From the Women's Health Initiative randomized controlled trial. JAMA 288(3):321-333. https://10.1001/jama.288.3.321

8. Hodis HN, Mack WJ, Henderson VW, Shoupe D, Budoff MJ, Hwang-Levine J, Li Y, Feng M et al (2016) Vascular Effects of Early versus Late Postmenopausal Treatment with Estradiol. N Engl J Med 374(13):1221-1231. https://10.1056/NEJMoa1505241

9. Mukund V, Mukund D, Sharma V, Mannarapu M, Alam A (2017) Genistein: Its role in metabolic diseases and cancer. Crit Rev Oncol Hematol 119:13-22. https://10.1016/j.critrevonc.2017.09.004

10. Schreihofer DA, Oppong-Gyebi A (2019) Genistein: mechanisms of action for a pleiotropic neuroprotective agent in stroke. Nutr Neurosci 22(6):375-391. https://10.1080/1028415X.2017.1391933

11. Thangavel P, Puga-Olguin A, Rodriguez-Landa JF, Zepeda RC (2019) Genistein as Potential Therapeutic Candidate for Menopausal Symptoms and Other Related Diseases. Molecules 24(21). https://10.3390/molecules24213892 
12. Prossnitz ER, Barton M (2011) The G-protein-coupled estrogen receptor GPER in health and disease. Nat Rev Endocrinol 7(12):715-726. https://10.1038/nrendo.2011.122

13. Zhang Z, Qin P, Deng Y, Ma Z, Guo H, Guo H, Hou Y, Wang S et al (2018) The novel estrogenic receptor GPR30 alleviates ischemic injury by inhibiting TLR4-mediated microglial inflammation. J Neuroinflammation 15(1):206. https://10.1186/s12974-018-1246-x

14. Lamkanfi M, Dixit VM (2014) Mechanisms and functions of inflammasomes. Cell 157(5):10131022. https://10.1016/j.cell.2014.04.007

15. Sharma D, Kanneganti TD (2016) The cell biology of inflammasomes: Mechanisms of inflammasome activation and regulation. J Cell Biol 213(6):617-629. https://10.1083/jcb.201602089

16. Kelley N, Jeltema D, Duan Y, He Y (2019) The NLRP3 Inflammasome: An Overview of Mechanisms of Activation and Regulation. Int J Mol Sci 20(13). https://10.3390/ijms20133328

17. Wang S, Wang J, Wei H, Gu T, Wang J, Wu Z, Yang Q (2020) Genistein Attenuates Acute Cerebral Ischemic Damage by Inhibiting the NLRP3 Inflammasome in Reproductively Senescent Mice. Front Aging Neurosci 12:153. https://10.3389/fnagi.2020.00153

18. St-Pierre J, Drori S, Uldry M, Silvaggi JM, Rhee J, Jager S, Handschin C, Zheng K et al (2006) Suppression of reactive oxygen species and neurodegeneration by the PGC-1 transcriptional coactivators. Cell 127(2):397-408. https://10.1016/j.cell.2006.09.024

19. Rius-Perez S, Torres-Cuevas I, Millan I, Ortega AL, Perez S (2020) PGC-1alpha, Inflammation, and Oxidative Stress: An Integrative View in Metabolism. Oxid Med Cell Longev 2020:1452696. https://10.1155/2020/1452696

20. Wrann CD, White JP, Salogiannnis J, Laznik-Bogoslavski D, Wu J, Ma D, Lin JD, Greenberg ME et al (2013) Exercise induces hippocampal BDNF through a PGC-1alpha/FNDC5 pathway. Cell Metab 18(5):649-659. https://10.1016/j.cmet.2013.09.008

21. Han B, Jiang W, Cui P, Zheng K, Dang C, Wang J, Li H, Chen L et al (2021) Microglial PGC-1alpha protects against ischemic brain injury by suppressing neuroinflammation. Genome Med 13(1):47. https://10.1186/s13073-021-00863-5

22. Idris Al (2012) Ovariectomy/orchidectomy in rodents. Methods Mol Biol 816:545-551. https://10.1007/978-1-61779-415-5_34

23. Mclean AC, Valenzuela N, Fai S, Bennett SA (2012) Performing vaginal lavage, crystal violet staining, and vaginal cytological evaluation for mouse estrous cycle staging identification. J Vis Exp 67e4389. https://10.3791/4389

24. Cai Y, Guo H, Fan Z, Zhang X, Wu D, Tang W, Gu T, Wang S et al (2020) Glycogenolysis Is Crucial for Astrocytic Glycogen Accumulation and Brain Damage after Reperfusion in Ischemic Stroke. iScience 23(5):101136. https://10.1016/j.isci.2020.101136

25. Bouet V, Boulouard M, Toutain J, Divoux D, Bernaudin M, Schumann-Bard P, Freret T (2009) The adhesive removal test: a sensitive method to assess sensorimotor deficits in mice. Nat Protoc 4(10):1560-1564. https://10.1038/nprot.2009.125 
26. Garcia JH, Wagner S, Liu KF, Hu XJ (1995) Neurological deficit and extent of neuronal necrosis attributable to middle cerebral artery occlusion in rats. Statistical validation. Stroke 26(4):627-634., 635. https://10.1161/01.str.26.4.627

27. Fernandes-Alnemri T, Wu J, Yu JW, Datta P, Miller B, Jankowski W, Rosenberg S, Zhang J et al (2007) The pyroptosome: a supramolecular assembly of ASC dimers mediating inflammatory cell death via caspase-1 activation. Cell Death Differ 14(9):1590-1604. https://10.1038/sj.cdd.4402194

28. Duan X, Li Y, Xu F, Ding H (2021) Study on the neuroprotective effects of Genistein on Alzheimer's disease. Brain Behav 11(5):e2100. https://10.1002/brb3.2100

29. Liang HW, Qiu SF, Shen J, Sun LN, Wang JY, Bruce IC, Xia Q (2008) Genistein attenuates oxidative stress and neuronal damage following transient global cerebral ischemia in rat hippocampus. Neurosci Lett 438(1):116-120. https://10.1016/j.neulet.2008.04.058

30. Aras AB, Guven M, Akman T, Alacam H, Kalkan Y, Silan C, Cosar M (2015) Genistein exerts neuroprotective effect on focal cerebral ischemia injury in rats. Inflammation 38(3):1311-1321. https://10.1007/s10753-014-0102-0

31. Rahman MM, Hongsprabhas $P$ (2016) Genistein as antioxidant and antibrowning agents in in vivo and in vitro: A review. Biomed Pharmacother 82:379-392. https://10.1016/j.biopha.2016.05.023

32. Donzelli A, Braida D, Finardi A, Capurro V, Valsecchi AE, Colleoni M, Sala M (2010) Neuroprotective effects of genistein in Mongolian gerbils: estrogen receptor-beta involvement. J Pharmacol Sci 114(2):158-167. https://10.1254/jphs.10164fp

33. Schreihofer DA (2005) Transcriptional regulation by phytoestrogens in neuronal cell lines. Mol Cell Endocrinol 231(1-2):13-22. https://10.1016/j.mce.2004.12.006

34. Du ZR, Feng XQ, Li N, Qu JX, Feng L, Chen L, Chen WF (2018) G protein-coupled estrogen receptor is involved in the anti-inflammatory effects of genistein in microglia. Phytomedicine 43:11-20. https://10.1016/j.phymed.2018.03.039

35. Jayaraj RL, Azimullah S, Beiram R, Jalal FY, Rosenberg GA (2019) Neuroinflammation: friend and foe for ischemic stroke. J Neuroinflammation 16(1):142. https://10.1186/s12974-019-1516-2

36. Miao EA, Leaf IA, Treuting PM, Mao DP, Dors M, Sarkar A, Warren SE, Wewers MD et al (2010) Caspase-1-induced pyroptosis is an innate immune effector mechanism against intracellular bacteria. Nat Immunol 11(12):1136-1142. https://10.1038/ni.1960

37. Cheng CF, Ku HC, Lin H (2018) PGC-1alpha as a Pivotal Factor in Lipid and Metabolic Regulation. Int J Mol Sci 19(11). https://10.3390/ijms19113447

\section{Figures}

\section{Figure 1}


Genistein upregulated the expression of GPR30 in the ischemic penumbra after I/R.

a. Western blotting showed that GPR30 expression was downregulated after I/R. b. Genistein increased the expression of GPR30 in the ischemic penumbra on Day 3 after reperfusion. c. The mRNA level of GPR30 was measured by RT-qPCR after l/R. d. GPR30 mRNA levels in the ischemic penumbra on Day 3 after reperfusion in the presence of genistein treatment. e. Representative images of immunofluorescence staining with antibodies against GPR30 and NeuN in the ischemic penumbra. f. Percentage of GPR30+NeuN+ cells relative to GPR30+ cells. g. Quantitative analysis of the relative fluorescence intensity of GPR30 in neurons. Double immunofluorescence staining revealed that genistein increased the expression of GPR30 in neurons on Day 3 after reperfusion. The data are presented as the mean \pm SD, $\mathrm{n}=4$. a,c: ${ }^{*} P<0.05$ vs. the Sham group; $\triangle P<0.05$ vs. the $1 \mathrm{~d}$ after I/R group; ${ }^{\#} P<0.05$ vs. the $3 \mathrm{~d}$ after $\mathrm{I} / \mathrm{R}$ group. b, d, g: ${ }^{*} P<0.05$ vs. the Vehicle group.

\section{Figure 2}

\section{GPR30 participated in the neuroprotective effect of genistein against reperfusion injury.}

a. Representative images of TTC staining of mouse brains at $7 \mathrm{~d}$ after l/R. b. The infract volumes, as measured by TTC staining. c. The adhesive removal test was performed to analyze somatosensory deficits before (Day -1) and after reperfusion. d-e. The total steps (d) and foot fault ratios (e) in the gridwalking test before (Day -1) and after reperfusion. The data are presented as the mean $\pm S D, n=8$. ${ }^{*}<$ 0.05 the Gen group vs. the Vehicle group; ${ }^{\#} P<0.05$ the Gen + GPR30 virus group vs. the Gen + Control virus group.

\section{Figure 3}

\section{GPR30 was involved in the antiapoptotic effect of genistein during I/R.}

a. Representative TUNEL staining in the ischemic penumbra on Day 3 after reperfusion. $b$. The percentage of TUNEL-positive neurons in the ischemic penumbra. c. Western blot analysis of cleaved caspase-3 expression in the ischemic penumbra $3 \mathrm{~d}$ after reperfusion. The upper panel shows cleaved caspase- 3 and the corresponding GAPDH bands. The histogram in the lower panel shows the densitometric analysis results. The data are presented as the mean $\pm S D, n=4 .{ }^{*} P<0.05$ the Gen group vs. the vehicle group; ${ }^{\#} P<0.05$ the Gen + GPR30 virus group vs. the Gen + Control virus group.

\section{Figure 4}


GPR30 mediated the inhibitory effect of genistein on the formation of NLRP3 inflammasomes in neurons.

a. Western blot analysis of NLRP3 expression in the ischemic penumbra at $3 \mathrm{~d}$ after reperfusion. The upper panel shows NLRP3 and the corresponding GAPDH bands. The histogram in the lower panel shows the densitometric analysis results. b. Western blot analysis of pro-caspase- 1 expression in the ischemic penumbra at $3 \mathrm{~d}$ after reperfusion. The upper panel shows pro-caspase- 1 and the corresponding GAPDH bands. The histogram in the lower panel shows the results of densitometric analysis. c. The mRNA level of NLRP3 was determined by RT-qPCR. $d$. The mRNA level of pro-caspase- 1 was determined by RT-qPCR. e. Coronal immunofluorescence images of the ischemic penumbra after staining with antibodies against NeuN and NLRP3. Neurons are marked with NeuN. f. The percentage of NLRP3+NeuN+ cells relative to NLRP3+ cells. g. Quantitative analysis of the relative fluorescence intensity of NLRP3 in neurons. Double immunofluorescence staining revealed that genistein decreased the expression of GPR30 in neurons on Day 3 after reperfusion, and this inhibitory effect was offset by the downregulation of GPR30. The data are presented as the mean $\pm \mathrm{SD}, \mathrm{n}=4$. ${ }^{\star} P<0.05$ the Gen group vs. the Vehicle group; ${ }^{\#} P<0.05$ the Gen + GPR30 virus group vs. the Gen + Control virus group.

\section{Figure 5}

\section{Neuronal GPR30 was involved in genistein-mediated inhibition of NLRP3 inflammasome activation and the release of inflammatory cytokines.}

a. The interaction (red particles) of NLRP3 with ASC was examined by Duolink analysis and DAPI (blue) counterstaining of nuclei. b. The quantified results of the Duolink analysis. c. Caspase-1 activity was measured by ELISA at $3 \mathrm{~d}$ after reperfusion in the ischemic penumbra. $\mathrm{d}-\mathrm{g}$. The levels of the proinflammatory cytokines IL-6, IL-1 $\beta$, TNF-a, and IL-18 in the ischemic penumbra were measured by ELISA at $3 \mathrm{~d}$ after reperfusion. The data are presented as the mean $\pm S D, a, b: n=4 ; c-g: n=5$. ${ }^{\star} P<0.05$ the Gen group vs. the Vehicle group; ${ }^{\#} P<0.05$ the Gen + GPR30 virus group vs. the Gen + Control virus group.

\section{Figure 6}

\section{Genistein regulated the expression of PGC-1a through GPR30 in neurons.}

a. Immunoblotting was used to measure the total protein level of PGC-1a after I/R in the penumbra. The relative optical density was calculated by dividing the density of the PGC-1 a band by that of the corresponding GAPDH band. b. Immunoblotting was used to measure the intranuclear protein level of PGC-1a after I/R in the penumbra. The relative optical density was calculated by dividing the density of the PGC-1a band by that of the corresponding histone band. c. The mRNA level of PGC-1a was determined by RT-qPCR. d. Quantitative analysis of the relative fluorescence intensity of PGC-1a in 
neurons. e. Representative immunofluorescence images showing staining with antibodies against NeuN and PGC-1a in the ischemic penumbra. The data are presented as the mean $\pm \mathrm{SD}, \mathrm{n}=4 .{ }^{\star} P<0.05$ the Gen group vs. the Vehicle group; ${ }^{\#} P<0.05$ the Gen + GPR30 virus group vs. the Gen + Control virus group.

\section{Figure 7}

\section{Neuron-specific PGC-1a knockdown promoted the transcription and expression of NLRP3.}

a. Representative immunofluorescence images showing staining with antibodies against NeuN and NLRP3 in the ischemic penumbra. b. Western blot analysis showed that PGC-1a knockdown significantly improved the expression of NLRP3. c. Western blot analysis of pro-caspase-1 expression at $3 \mathrm{~d}$ after reperfusion in the ischemic penumbra. The upper panel shows pro-caspase- 1 and the corresponding GAPDH bands. The histogram in the lower panel shows the densitometric analysis results. $d$. The mRNA level of NLRP3 was determined by RT-qPCR. e. The mRNA level of pro-caspase- 1 was determined by RTqPCR. $f$. The percentage of NLRP3+NeuN+ cells relative to NLRP3+ cells. g. Quantitative analysis of the relative fluorescence intensity of NLRP3 in neurons. The data are presented as the mean $\pm S D, n=4 .{ }^{*} P<$ 0.05 vs. the Gen + Control virus group.

\section{Figure 8}

\section{Neuron-specific PGC-1a knockdown promoted the activation of NLRP3 inflammasomes and the release of downstream inflammatory cytokines.}

a. Duolink analysis showed the interaction (red particles) of NLRP3 with ASC, and DAPI (blue)counterstained nuclei. b. Quantitative analysis of the interaction of NLRP3 with ASC. c. Caspase-1 activity in the penumbra was examined by ELISA at $3 \mathrm{~d}$ after reperfusion. $\mathrm{d}-\mathrm{g}$. The levels of the proinflammatory cytokines IL-6, TNF-a, IL-1 $\beta$ and IL-18 in the penumbra were examined by ELISA at $3 \mathrm{~d}$ after reperfusion. The data are presented as the mean $\pm S D, n=5 . * P<0.05$ vs. the Gen + Control virus group.

\section{Figure 9}

\section{Neuron-specific PGC-1a knockdown exacerbated ischemic injury and apoptosis.}

a. Representative TTC staining at $3 \mathrm{~d}$ after l/R. b. The infract volumes, as shown by TTC staining. c. The adhesive removal test was performed to analyze somatosensory deficits before (Day-1) and after reperfusion. d-e. The total steps (d) and foot fault ratios (e) in the grid-walking test before (Day-1) and after reperfusion. f. Representative TUNEL staining in the ischemic penumbra on Day 3 after reperfusion. 
g. The percentage of TUNEL-positive neurons in the ischemic penumbra. h. Western blot analysis was performed to measure cleaved caspase-3 expression in the ischemic penumbra at $3 \mathrm{~d}$ after reperfusion. The upper panel shows cleaved caspase- 3 and the corresponding GAPDH bands. The histogram in the lower panel shows the densitometric analysis results. The data are presented as the mean \pm SD, a-e: $n=8$; $\mathrm{f}-\mathrm{h}: \mathrm{n}=4 .{ }^{*} P<0.05$ vs. the Gen + Control virus group.

Figure 10

Schematic diagram of the GPR30/PGC-1a/NLRP3 pathway in genistein-mediated neuroprotection against reperfusion injury after ischemic stroke.

\section{Supplementary Files}

This is a list of supplementary files associated with this preprint. Click to download.

- Supplementaryfigure.docx 